\title{
A NOVEL INDEX STRUCTURE FOR LARGE SCALE IMAGE DESCRIPTOR SEARCH
}

\author{
Jiangbo Yuan, Xiuwen Liu \\ Florida State University \\ Department of Computer Science \\ Tallahassee, FL, USA
}

\begin{abstract}
This paper presents a $k$-means based algorithm for approximate nearest neighbor search. The proposed Embedded $k$-Means algorithm is a two-level clustered index structure which consists of two groups of centroids; additionally, an inverted file is used for recording of the assignments. The coarse-to-fine structure achieves high search efficiency using multi-assignment operations on the coarse level. At the query stage, pruning strategies are utilized to balance the trade-off between search qualities and speeds. Our algorithm is able to explore the neighborhood space of a given query efficiently. Due to its good recall/selectivity and memory efficiency, the proposed algorithm is scalable and is able to process very large databases. Experimental results on SIFT and GIST image descriptor datasets show search performance better and comparable to the state-of-the-art approaches with lower memory usage and complexity.
\end{abstract}

Index Terms $-k$-means, approximate nearest neighbor search, multi-assignment, pruning strategies, image descriptor indexing

\section{INTRODUCTION}

Fast nearest neighbor search in high-dimensional space is a fundamental problem for large scale content based image/video/audio retrieval systems in computer vision and multimedia communities. The scalability and accuracy of traditional nearest neighbor search algorithms are severely limited by the curses of dimensionality for data in high dimensional. As a result, real-world applications, where high dimensional data are ubiquitous, require new approaches and algorithms to overcome the limitations in needed computation time, index storage, and search quality. Recently, Approximate Nearest Neighbor (ANN) search approaches on image descriptors (SIFT [1] and GIST [2]) have demonstrated to be effective in handle largescale image retrieval or other similar problems.

In the last decade, Local Sensitive Hashing (LSH) is one of the best known ANN methodologies. Its variants (e.g., [3, 4]) have been applied in different applications; however, LSH algorithms often require large memory usage. To overcome the memory limitation, algorithms which use limited memory (within memory-based index structures) are being proposed since they provide a better solution to meet the requirements of large scale real-time search systems. Those algorithms are often designed with more consideration of data distributions (i.e., Multiple KD-trees [5], Hamming Embedding [6], Hierarchical $k$-Means Trees [7, 8] and Product Quantization [9, 10]).

We introduce a new coarse-to-fine clustering-based index structure, Embedded $k$-Means (EMKM), which combines with multiassignment and pruning strategies to enable exploring of the limited neighborhood space while achieves most true neighbors of a given query. By performing clustering in the residual space [9],
EMKM forms a virtual tree-structure similar to hierarchical $k$-means but using an uniform clustering on the second level so with limited centroids/reference points. At the same time, performing multiassignments on the first level increases the probabilities to have the nearest neighbors falling into the same cells while the embedded clustering avoids to fully visiting those enlarged cells (see Fig. 1). Moreover, the pruning strategy limits the number of candidate points for exhaustive search to balance the search speeds and search qualities.

The remainder of the paper is organized as follows. Section 2 introduces our new ANN algorithm in details. Section 3 discusses performance comparison between our approach and several state of the art methods, then provides certain experimental results on large SIFT and GIST datasets.

\section{EMBEDDED $K$-MEANS}

The index structure of Embedded $k$-Means consists of two parts. One of them contains two level of centroids and another one is of inverted file. The inverted file, which is used for recording of the clustered point groups, is built up in the same way as [7] to fit to the hierarchical structure.

\subsection{Index structure construction}

The EMKM index structure is mainly constructed by the following steps:

1. cluster all database points by $k$-means to get the first level coarse centroids, refer to $k=k_{1}$;

2. assign each database point to multiple, say $m_{\mathrm{a}}$, of its nearest coarse centroids;

3. mix all the residual data points (by subtracting each of the assigned coarse centroids) and to be clustered by $k$-means, which forms a second level fine centroids; refer to $k=k_{2}$;

4. assign each residual data point to its nearest fine centroid.

Then we get $k_{1}$ centroids from the first level and $k_{2}$ from the second level, after embedding the $k_{2}$ residual centroids into every coarse centroid, finally we get in total $k_{1} \times k_{2}$ cells in the Inverted File. Given a database with $N$ points in $D$ dimensions, however, the true storage for centroids is only $\left(k_{1}+k_{2}\right) \times D$. Fig. 1 is an illustration of this procedure. We use $k$-means to cluster those $2 D$ random points while represent the clusters by the Vorono $i$ cells.

Multiple assignment One of the limitations of $k$-means based ANN methods is that the choosing $k$ often suffers the trade-off between recall and precision. In other words, a larger $k$ is wanted because it gives finer clustering and filters more points out for further processing. However, it retains fewer target nearest neighbors 

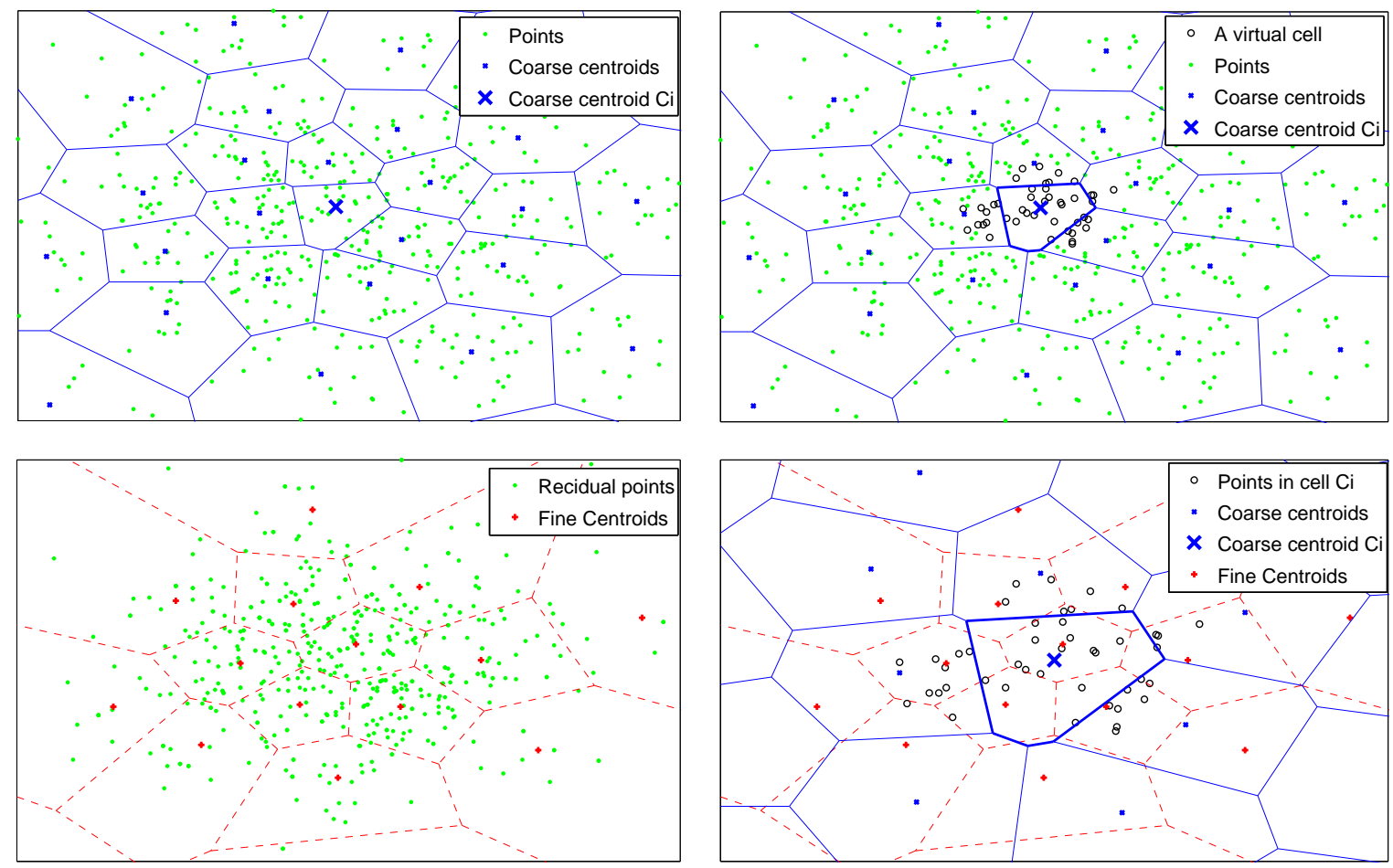

Fig. 1. Overview of the index construction procedure. Top left figure shows all points and coarse cells; we pick out one cell $C_{\mathrm{i}}$ to illustrate its new cover range after multiple assignment in the top right figure; black small circles are those data points assigned to centroid $C_{\mathrm{i}}$. Bottom left figure shows all residual points and fine cells; bottom right figure illustrates the coarse cell $C_{\mathrm{i}}$ with embedded fine cells.

in near cells that results in more cells have to be visited. In our approach, we assign each database point to $m_{\mathrm{a}}$ of its nearest centroids in the coarse level. But we must to notice that the multi-assignment strategy eventually enlarges the cover range of each single cell. It gives very limited advantage if no further precessing are involved. We apply a second level $k$-means clustering on the $N \times m_{\mathrm{a}}$ points (or randomly choosing a subset) in the residual space. (A residual point is calculated by subtracting the corresponding assigned centroid from a given data point.) Finally, each of the $N \times m_{\mathrm{a}}$ residual points are assigned to its nearest fine centroid. Successively, the assignment complexity is $\left(k_{1}+m_{\mathrm{a}} \times k_{2}\right) \times D$ for a single database point. Please see Fig. 2 and Fig. 3 that demonstrated the advantages of our multiple assignment approach on both running time and selectivity. The selectivity means the proportion of the number of points on the final short list which are ready for exhaustive checking.

\subsection{Query process}

Due to the multi-assignment, given a query $q$ whose coarse nearest centroid is $C_{\mathrm{i}}$ then it is actually assigned into the bold cell whose search range covers all the points in black circles in the fine level (see Fig. 1). In other words, the searching range in a single cell is larger than its possible assignment range for a given query $q$. Compared with hard assignment approach, this increases the probability for reaching $q$ 's near neighbors in a single cell. It is obviously that the multi-assignment still cannot guarantee the nearest neighbor of $q$ is located at its nearest coarse cell. Therefore, multi-probe operations are also employed in our approach for both two levels. How-

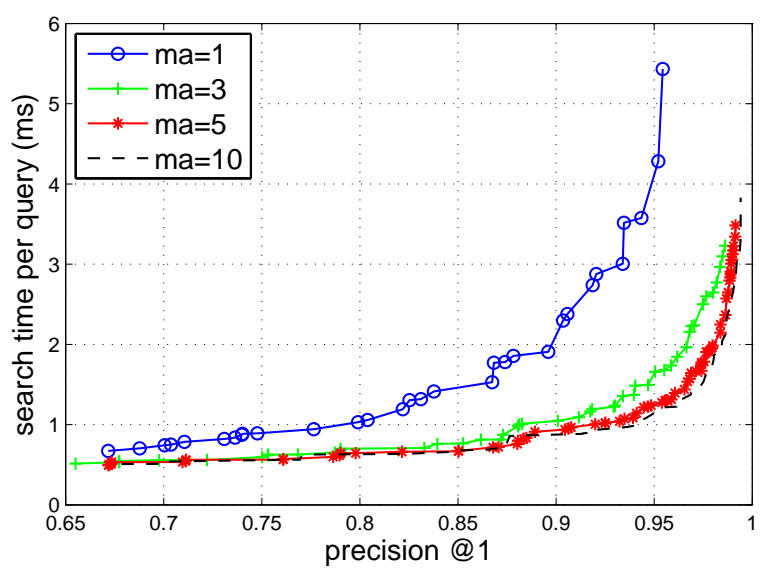

Fig. 2. Search speed performance on SIFT1M with parameters of $k_{1}=10000$ and $k_{2}=100$ and different $m_{\mathrm{a}}$ values.

ever, multi-assignment strategy significantly reduces the probe times have to be made in the coarse level which consequently reduces the operations made on the fine centroids.

Pruning strategies The pruning process consists of two steps. Firstly, we limit the amounts of visited cells on both coarse and fine levels. Assuming that in the coarse level we probe the query $q$ to $m p_{1}$ 


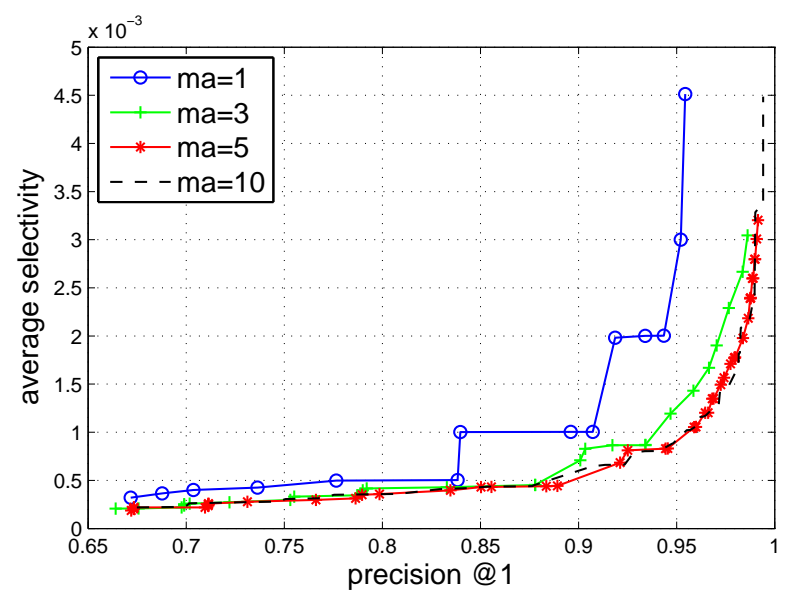

Fig. 3. Selectivity performance on SIFT1M with parameters of $k_{1}=$ 10000 and $k_{2}=100$ and different $m_{\mathrm{a}}$ values.

\begin{tabular}{lccc} 
Dataset & Database & Learning Set & Query Set \\
\hline SIFT1M & $1,000,000$ & $1,000,000$ & 10,000 \\
SIFT50M & $50,000,000$ & $1,000,000$ & 10,000 \\
SIFT100M & $100,000,000$ & $1,000,000$ & 10,000 \\
GIST1M & $1,000,000$ & 500,000 & 1,000 \\
\hline
\end{tabular}

Table 1. Datasets used in this paper.

cells. For each probe, we assign the new residual query to a fixed number $m p_{2}$ fine cells. Thus each probe collects some candidate neighbors which are "surrounding" to $q$. Finally, the coarse short list is constructed by sorting all the candidates cells based on distances.

However, for different queries, the surrounding populations of near cells are changing due to the diversity of the distribution densities. Thus the choices of $m p_{1}$ and $m p_{2}$ are often biased due to some difficult queries which require to expand more cells. Nonetheless, we added in another pruning strategy which checks the amount of candidate points based on the results after the first pruning step. After setting up a maximal visit number $N_{\text {maxvisit }}$, the visited number of cells are not necessary to be the same for different queries. This pruning strategy significantly speeds up the searching while with limited loss of accuracy. It actually estimates the surrounding distribution density of a given query and enables a fast fetching of its compact neighborhood space relying on the very fine clustering. mately

Eventually, the search complexity for a given query is approxi-

$$
\left(k_{1}+m p_{1} \times k_{2}+N_{\text {maxvisit }}\right) \times D .
$$

\section{EXPERIMENTAL RESULTS}

Datasets Recently, some very large image descriptor datasets are published to provide a baseline for evaluation on different ANN algorithms. For instance, [9] and [10] introduced packages collected several SIFT (128 dimensions) datasets in scales up to 1 billion and GIST1M with 1 million GIST descriptor vectors in 960 dimensions. Table 1 gives details of datasets we used in this paper including database, learning set and query set sizes. All SIFT datasets are subsets from the package of big ANN SIFT1B [10].

\begin{tabular}{lcc} 
Algorithm & $\begin{array}{c}\text { Centroids } \\
\text { (complexity) }\end{array}$ & $\begin{array}{c}\text { Inverted File } \\
\text { (memory in bits) }\end{array}$ \\
\hline$k$-means & $k D$ & $N\left\lceil\log _{2} N\right\rceil$ \\
HKM & $\frac{b_{\mathrm{f}}}{b_{\mathrm{f}}-1}(k-1) D$ & $N\left\lceil\log _{2} N\right\rceil$ \\
PKM & $m k^{*} D^{*}=k^{*} D$ & $N\left(\left\lceil\log _{2} N\right\rceil+m \log _{2} k^{*}\right)$ \\
EMKM & $\left(k_{1}+k_{2}\right) D$ & $m_{\mathrm{a}} N\left\lceil\log _{2} N\right\rceil$ \\
\hline
\end{tabular}

Table 2. Memory usage complexities of different index structures.

\subsection{Empirical evaluation}

In this section we discuss how to configure good parameters in our approach. $\left\{k_{1}, k_{2}, m_{\mathrm{a}}\right\}$ impact on both index structure and query process while $\left\{m p_{1}, m p_{2}, N_{\text {maxvisit }}\right\}$ are purely for query process. Empirically, $k_{1} \times k_{2} \cong N$ is a good choice since it gives an approximate population $p_{\text {cell }}=m_{\mathrm{a}}$ for a single cell which is very fine but not too sparse. And $k_{1}$ can be slightly larger than $k_{2}$ considering the multi-probe complexity. At the same time, $N_{\text {maxvisit }}$ is used as a threshold to control the range of expansion. As the increases of $m p_{1}$ and $m p_{2}$, both of the search accuracy and selectivity will get stuck in a "upper bound" after the shortlist from the pruning stage one overflows. Consequently, it is reasonable to set up the estimated size of shortlist $\left(m p_{1} \times m p_{2} \times p_{\text {cell }}\right)$ from the pruning stage one to be slightly larger than $N_{\text {maxvisit }}$.

\subsection{Comparison with the state of the arts}

Memory usage Jegou et al. [9] report that for SIFT 1 million data set, the index structure of FLANN [8] requires more than $250 \mathrm{MB}$ of memory while for IVFADC [9] it occupies less than $25 \mathrm{MB}$. It is also reported in [7] that the index structure for their hierarchical $k$-means tree structure requires $143 \mathrm{MB}$ for a tree with 1 million leaf nodes (leaf centroids). However, our method only needs with less than $4.9 \mathrm{MB}\left(k_{1}=10000\right.$ and $\left.k_{2}=100\right)$ for centroid file and less than $23 \mathrm{MB}$ (with $m_{\mathrm{a}}=5$ ) or $15 \mathrm{MB}$ (with $m_{\mathrm{a}}=3$ ) for the inverted file. The memory usage in total is comparable to IVFADC and much more efficient than other similar tree structuring methods. Table 2 reports separately the memory usage complexities of centroids and inverted file while Table 3 gives the total amount of cells achieved and assignment complexity for several $k$-means based approaches. HKM represents Hierarchical $k$-Means and PKM is Product $k$-Means or IVFADC in [9].

Search performance Referring to the comparison between IVFADC and FLANN in the paper [9] on SIFT 1 million dataset, we can see that our EMKM approach (refer to Fig. 2) achieves slightly better performance than IVFADC. Moreover, FLANN (including KD-trees and hierarchical k-means trees) is apparently outperformed by both EMKM and IVFADC. Our experimental environment setup is very close to Jegou et al. did in their paper [9] since we use the same YAEL library for fundamental calculations and all results were produced by similar single-core machines. The evaluation metric precision@1 is consistent with 1-recall@1 in [9] and precision in [8].

\subsection{Large-scale experiments}

Searching on large-scale SIFT datasets (SIFT50M and SIFT100M) are performed in order to evaluate the scalability and the efficiency of EMKM (see Fig. 4). We achieved about $96 \%$ precision in average of $8 \mathrm{~ms}$ per query on SIFT50M and about $92 \%$ precision in 


\begin{tabular}{lcc} 
Algorithm & Total Cells & Assignment Complexity \\
\hline$k$-means & $k$ & $k D$ \\
HKM & $b_{\mathrm{f}}^{l}=k$ & $b_{\mathrm{f}} l D$ \\
PKM & $\left(k^{*}\right)^{m}=k$ & $k^{*} D$ \\
EMKM & $k_{1} k_{2}=k$ & $\left(k_{1}+m_{\mathrm{a}} k_{2}\right) D$ \\
\hline
\end{tabular}

Table 3. Assignment complexity of different index structures.

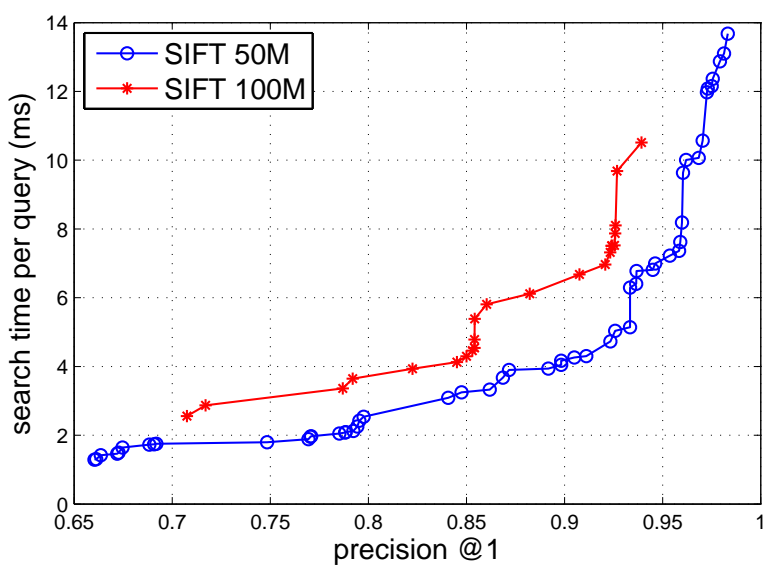

Fig. 4. Search performance on datasets SIFT50M and SIFT100M with parameters of $k_{1}=10000, k_{2}=1000$ and $m_{\mathrm{a}}=5$.

around $7 m s$ on SIFT100M. The global descriptors such as GIST take advantage of relatively lower dimensional representation of a single image and they are more attractive for scene recognition, webscale image search or copy detection tasks $[11,12]$. For GIST1M, we achieved about $95 \%$ precision took less than $60 \mathrm{~ms}$ (see Fig. 5). Compared to other methods, our approach is especially interested for high-precision search solutions.

\section{CONCLUSION}

Through comparison with the state of the art methods on both memory usage and search efficiency, we believe that the new proposed EMKM index structure within multiple assignment and pruning strategies is a promising scheme to handle searching with very large databases of image descriptors. In the future, it is possible to extend the current two level index structures to multi-levels in order to enable better performance; and a multiple tree structure could be considered in the way of randomized KD-trees does. As an indexing structure, it is also possible to combine it with the different kinds of emerging descriptor coding techniques to improve on both speed and storage .

\section{REFERENCES}

[1] David G. Lowe, "Distinctive image features from scaleinvariant keypoints," IJCV, vol. 60, pp. 91-110, November 2004.

[2] Aude Oliva and Antonio B. Torralba, "Modeling the shape of

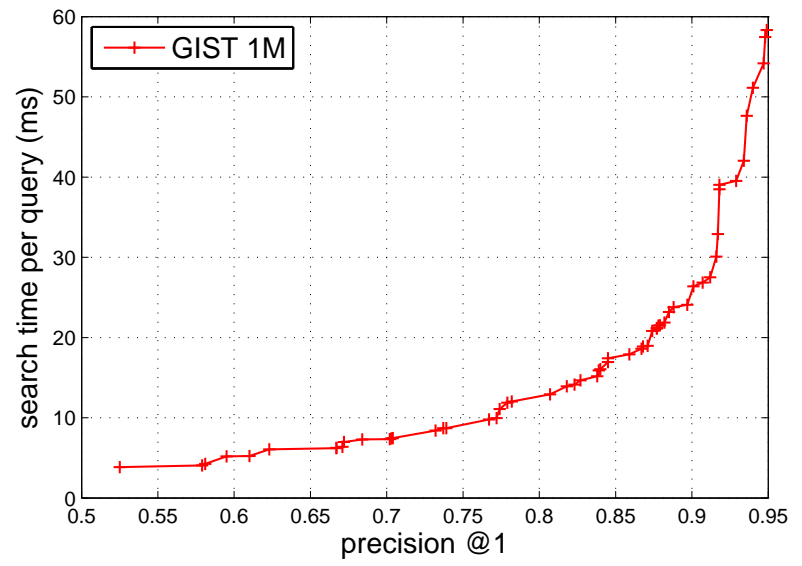

Fig. 5. Search performance on GIST1M dataset with parameters of $k_{1}=1000, k_{2}=1000$ and $m_{\mathrm{a}}=5$.

the scene: A holistic representation of the spatial envelope," IJCV, vol. 42, pp. 145-175, 2001.

[3] Mayur Datar and Piotr Indyk, "Locality-sensitive hashing scheme based on p-stable distributions," in $S C G^{\prime} 04$. 2004, pp. 253-262, ACM Press.

[4] Alexandr Andoni, "Near-optimal hashing algorithms for approximate nearest neighbor in high dimensions," in FOCSO6. 2006, pp. 459-468, IEEE Computer Society.

[5] Chanop Silpa-Anan and Richard Hartley, "Optimised kd-trees for fast image descriptor matching," in CVPR, 2008, pp. 1-8.

[6] Herve Jegou, Matthijs Douze, and Cordelia Schmid, "Hamming embedding and weak geometric consistency for large scale image search," in ECCV, Berlin, Heidelberg, 2008, ECCV '08, pp. 304-317, Springer-Verlag.

[7] D. Nister and H. Stewenius, "Scalable recognition with a vocabulary tree," in CVPR, 2006, vol. 2, pp. 2161-2168.

[8] Marius Muja and David G. Lowe, "Fast approximate nearest neighbors with automatic algorithm configuration," in $I C$ CVTA, VISSAPP'09, 2009, pp. 331-340.

[9] Hervé Jégou, Matthijs Douze, and Cordelia Schmid, "Product quantization for nearest neighbor search," PAMI, vol. 33, no. 1, pp. 117-128, Jan 2011.

[10] Hervé Jégou, Romain Tavenard, Matthijs Douze, and Laurent Amsaleg, "Searching in one billion vectors: re-rank with source coding," in ICASSP, Prague Czech Republic, May 2011, QUAERO.

[11] Antonio Torralba, Robert Fergus, and Yair Weiss, "Small codes and large image databases for recognition," in CVPR. 2008, IEEE Computer Society.

[12] Matthijs Douze, Hervé Jégou, Harsimrat Sandhawalia, Laurent Amsaleg, and Cordelia Schmid, "Evaluation of gist descriptors for web-scale image search," in CIVR, New York, NY, USA, 2009, CIVR '09, pp. 19:1-19:8, ACM. 